This is not the version of record. The published version can be found at: https://doi.org/10.1016/j.ajodo.2020.02.012

Crown Copyright @ 2021 All rights reserved.

This manuscript version is made available under the CC-BY-NC-ND 4.0 license http://creativecommons.org/licenses/by-nc-nd/4.0/" 


\section{Quantitative investigation of the aesthetic impact of lip prominence in relation to the E-line}

\section{Abstract}

Introduction: The purpose of this quantitative investigation was to assess the influence of lip prominence in relation to the E-line on perceived attractiveness and threshold values of desire for treatment.

Methods: The lip prominence of an idealized silhouette male Caucasian profile image was altered incrementally between -16 to $+4 \mathrm{~mm}$ from the E-line. The images were rated on a Likert scale by pretreatment orthognathic patients $(n=75)$, laypeople $(n=75)$ and clinicians $(n=35)$.

Results: In terms of perceived attractiveness, a lips to E-line distance within the ranges of -12 to $-16 \mathrm{~mm}$ and 0 to $+4 \mathrm{~mm}$ in relation to the E-line was associated with a reduction in median attractiveness scores to below 4 in the patient and clinician groups of observers; for the lay group, the corresponding ranges were -14 to $-16 \mathrm{~mm}$ and +2 to $+4 \mathrm{~mm}$. Relative lip prominence appears to be viewed as more attractive than lip retrusion. Clinicians were generally least likely to suggest treatment for varying levels of bilabial position. For a number of the images there was reasonable agreement among clinicians and lay people as to whether treatment is required. For the clinician group the only categories for desire for treatment were at a lip to E-line distance within the ranges of $-14 \mathrm{~mm}$ to $-16 \mathrm{~mm}$ and $+2 \mathrm{~mm}$ to $+4 \mathrm{~mm}$.

Conclusion: It is recommended that the range of normal variability of the prominence of the lips, as well as threshold values of the desire for treatment, be considered in planning.

\section{Keywords}

Bilabial prominence, lips, attractiveness perception, desire for treatment, E-line 


\section{INTRODUCTION}

The relative prominence or retrusion of lip position within the face is thought to be a significant facial aesthetic parameter. ${ }^{1}$ The prominence and/or size of the lips can be altered directly, for example by surgical reduction of the vermilion or alternatively lip augmentation with fillers; or indirectly, by sagittal movement of the maxillary and mandibular incisors, the associated dentoalveolus, or orthognathic surgery, which has a variable effect on lip prominence due to inherent soft tissue variability. ${ }^{2}$

For any given population, lip prominence will have an "average" value or "norm", specific for age, gender and ethnicity, and an associated range of normal variability, with appearance concerns often resulting from a significant deviation of the sagittal lip position from the accepted norm for the population. For clinical diagnosis and treatment planning, it is important to know at what point the deviation of lip prominence moves from an acceptable range of variability to being perceived as a facial deformity. The magnitude of the deviation, whether it is due to the underlying dentoalveolar framework, the soft tissue thickness or a combination of the two, is an important factor in deciding when orthodontic treatment and/or surgery may be required. If the magnitude of the discrepancy of bilabial prominence or retrusion is great, the treatment planning decision may be relatively straightforward. However, a significant number of patients are regarded as borderline in terms of need for treatment. In such patients, the decision-making process may be transferred from subjective clinical judgement to objective, evidence-based guidance based on the data from studies investigating perceptions of bilabial positional attractiveness.

One of the most well-known analyses for lip prominence is the 'esthetic line' (E-line) described by Ricketts over fifty years ago as part of his computerised cephalometric analysis. ${ }^{3,4}$ It is worth noting that the vast majority of the Ricketts analysis is dentoskeletal, but he also described the E-line (nose-chin line) as joining the tip of the nose and the most 
prominent point on the soft tissue chin in profile view, and based on his data derived from analysis of 1000 cases (600 females, 400 males) with an average age of "about nine years", provided the average value of the distance from the lower lip to the E-line as $-2 \mathrm{~mm}$. Positive values would be with the lower lip ahead of the E-line. There is considerable sagittal and vertical growth of the nose and lips after the age of nine years and as such, the lips will tend to move in a negative direction relative to the E-line. Ricketts conceded that considerable individual variation existed, but his general view was that the upper lip should be approximately $4 \mathrm{~mm}$ and the lower lip $2 \mathrm{~mm}$ behind the E-line in an average Caucasian adult. $^{4}$

As with any facial parameter, it is generally acknowledged that bilabial prominence has a range of normal individual variability. For comparative purposes and to assess potential similarities and differences, it is worthwhile to look at bilabial prominence in previous anthropometric, soft tissue cephalometric and attractiveness investigations. Although Farkas $^{5,6}$ provided average values for many facial parameters, based on anthropometric studies, for North American adults of white ethnicity, Chinese Americans and African Americans, unfortunately, he did not anthropometrically measure bilabial prominence in any of his investigations. However, a number of modern authorities have provided "ideal" values for the bilabial prominence, based either on anecdotal evidence and the "good eye" of the respective clinician, data from treated cases, or normative values from growth data. In their longitudinal cephalometric growth data Riolo et al. ${ }^{7}$ did not evaluate bilabial prominence, however, Bhatia and Leighton ${ }^{8}$ provided values of between -4 to $-6 \mathrm{~mm}( \pm 2 \mathrm{~mm})$ in adult males and approximately $-5 \mathrm{~mm}( \pm 2.5 \mathrm{~mm})$ in adult females for the upper lip, and for the lower lip -3 to $-4 \mathrm{~mm}( \pm 2 \mathrm{~mm})$ in adult males and -3 to $-3.5 \mathrm{~mm}( \pm 2 \mathrm{~mm})$ in adult females. Adults in their group ranged between 16 to 20 years of age. 
Coleman et al. ${ }^{9}$ assessed the influence of chin prominence on preferred lip position in profile view, and fuller lip positions were preferred for the more extreme retrognathic and prognathic profiles. More retrusive lip positions were preferred for the more average profiles and generally they found no differences between their three evaluator groups (patients, parents, and orthodontists) or between male and female evaluators. Czarnecki et al. ${ }^{10}$ constructed androgynous profile silhouettes graded for attractiveness by professionals and found that more lip protrusion was deemed acceptable for both male and female faces when either a large nose or a large chin was present. Foster ${ }^{11}$ had groups of orthodontists, dentists, art students, and lay people of different ethnic backgrounds select the most attractive profile from a set of profile silhouettes with their lip prominence altered in $2 \mathrm{~mm}$ increments from 6 to $+6 \mathrm{~mm}$. He found that the diverse groups seemed to share a common aesthetic standard for lip prominence, in most cases within 1-2 $\mathrm{mm}$, that all groups were consistent in assigning fuller lips for younger ages, that all groups preferred at least $3 \mathrm{~mm}$ fuller lips for adult females; except orthodontists, who saw full lips at $1.4 \mathrm{~mm}$, and all groups preferred adult male lips to be more behind the E-line than the original Ricketts "ideal" values, with adult females closer to, but still further behind the "ideal" values.

A number of previous studies have assessed sagittal lip prominence in relation to ethnic variability of the images and/or observers. Hall et al. ${ }^{12}$ assessed profile silhouettes of 30 African American and 30 white patients, ranging in age from 7 to 17 years, rated by white and African American orthodontists and lay people. They found that the participants preferred the African American sample with upper and lower lips that were more prominent compared with the white sample. However, only the choice of the African American orthodontists for the African American sample was significantly different for this parameter. They also found significant ethnic variation, with the white orthodontists giving the highest mean scores for the profile chosen, whereas the African American laypersons gave the lowest 
scores. McKoy-White et al. ${ }^{13}$ looked at lip protrusion and retrusion, resembling bimaxillary protrusion and retrusion, using manipulated photographs of three black female profiles. They found that white orthodontists preferred flatter profiles than the black women patients, and that black women patients preferred fuller profiles than the black orthodontists. They also found subjective differences in profile attractiveness among different ethnic groups, subjective differences in profile attractiveness among orthodontists of different ethnic groups (white or African American), and contrary to previous research, that black women did not favour a flatter or more Caucasian profile. Mejia-Maidl et al. ${ }^{14}$ assessed manipulated the lip prominence in photographs of two male and two female individuals of Mexican descent and found that Mexican Americans preferred upper and lower lip positions to be less protrusive than did white Caucasians. Battle et al. ${ }^{15}$ assessed lip prominence in African American profiles to see whether the perception of lip prominence in modern African Americans had changed. They found that younger orthodontists favored more prominent lips in profile and suggested that a new set of standards for lip prominence in this population is necessary to reflect the current trend in the concept of an attractive face. They concluded that orthodontists working on various ethnic populations should be more conscious of using standardized profile analyses. Huang et al. ${ }^{16}$ assessed 60 Chinese patients with bimaxillary protrusion and found that retraction of their lips relative to the E-line correlated with an improvement in attractiveness ratings.

In further identifying and attempting to understand ethnic differences, Al Abdulwahhab et al. ${ }^{17}$ assessed a Saudi population, finding that the Saudi observers generally preferred lips to be further behind the E-line than the Western standard, which they took as upper lip -3mm and lower lip -1mm behind the E-line. They also noted the observers' greater tolerance for the lips to move away from rather than towards the E-line. Interestingly, their initial standard of $-3 \mathrm{~mm}$ is similar to that suggested by Ricketts, ${ }^{4}$ though Ricketts' values were based on 
children prior to the adolescent growth of the nose and chin, which would inevitably move the E-line away from the lips. Chong et al. ${ }^{18}$ compared preferences of white Australian judges and Chinese judges based in China in relation to lip prominence. They found that the Chinese judges preferred a more retrusive profile and were more likely to rate prominent lips, particularly ahead of the E-line, as unacceptable, compared with the white judges. The ethnicity of the judges was found to be a significant factor influencing the perception of aesthetic lip prominence. Hockley et al. ${ }^{19}$ compared perceptions of attractiveness of African American profile photographs with silhouettes of the same images. They found that flatter profiles with less lip prominence than the aesthetic 'norm' were more often preferred in the silhouettes than in the photographs and concluded that using silhouettes to evaluate patient aesthetics could influence clinicians or researchers to select profiles that are flatter than the established aesthetic 'norm'. The small number of raters ( $n=15$, all faculty and residents) in this investigation warrants a repeat of these potentially interesting results.

The principal aim of this investigation was to evaluate quantitatively the influence of lip prominence as represented by sagittal position in relation to the E-line on perceived attractiveness. The relationship between lip prominence and attractiveness was recorded to ascertain the range of normal variability in terms of observer acceptance, and to determine the clinically significant threshold value or cut-off point beyond which, lip position is perceived as unattractive and treatment is desired. The perceptions of patients, clinicians and laypeople were compared for these different variables.

\section{Materials and methods}

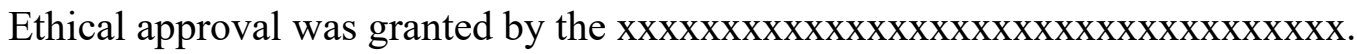

Two-dimensional profile silhouettes are used routinely to assess perception of facial attractiveness. $^{20,21}$ A profile silhouette image was created with computer software (Adobe Photoshop CS2, San Jose, California). The image was manipulated using the same software 
to construct an "ideal" profile image with proportions and linear and angular soft-tissue measurements, ${ }^{5,6,22}$ based on currently accepted criteria for an idealized Caucasian male profile, as previously described. ${ }^{21}$ The sagittal position of the lips of the idealized image (image BE: -6 mm measured as upper lip from E-line) was altered in 2-mm increments from 16 to $+4 \mathrm{~mm}$ to represent variations of bilabial position and morphology from excessive prominence to excessive retrusion (Figure 1).

Based on the results of a pilot study and power calculation, 185 observers took part in the study, separated into three groups (pre-treatment orthognathic patients, laypeople and clinicians; Table 1) with the following selection criteria:

1. Patients: pretreatment (only one consultation appointment); primary concern was facial appearance; no previous facial surgical treatment; no history of facial trauma; no severe psychological issues (based on patient interview by lead investigator);

2. Laypeople: no previous facial surgery, deformities, or history of facial trauma; selected randomly from a range of non-medical backgrounds (e.g. teachers, teaching assistants, parents of school children, police officers, firefighters); and

3. Clinicians: involved in the management of patients with facial deformities; included 19 maxillofacial surgeons and 16 orthodontists, with 1 to 16 years of experience in the clinical management of patients requiring orthognathic and facial reconstructive surgery.

Each observer was given a questionnaire and asked to provide the following information: (1) age, (2) gender, (3) ethnic origin (white or non-white), (4) how would you rate the attractiveness of your facial appearance? and (6) how important do you think it is to have an attractive facial appearance? An instruction sheet accompanied the questionnaire, asking the observers to rate each image in terms of facial attractiveness using the following rating scale: 
1. Extremely unattractive

2. Very unattractive

3. Slightly unattractive

4. Neither attractive nor unattractive

5. Slightly attractive

6. Very attractive

7. Extremely attractive

Observers were also asked whether they would consider treatment to correct the appearance if this was their facial appearance (yes or no).

The images were placed in random order into the software application Microsoft PowerPoint (Microsoft Corporation, Redmond, WA). Each image was identified by a randomly assigned double letter in the top right corner of the screen (e.g. BE and CF; Figure 2). A duplicate image assessed intra-examiner reliability (images DH and EG). Each observer sat undisturbed in the same room in front of the same computer and 17-in. flat screen monitor. The presentation and the images were created in such a way that each of the profile silhouette images, when viewed on the monitor, had the same dimensions as a normal human head, based on an average lower facial height, reducing the potential effect of image size on observer perception. Each observer examined the images in the PowerPoint presentation by pressing the "Page Down" button on the keyboard, in their own time. The Likert-type rating scale is largely accepted in the psychology literature as the most useful rating method. ${ }^{23}$ The seven-point Likert scale was used by each observer to rate each image in terms of attractiveness.

\section{Statistical analysis}

The median and interquartile observer ratings were calculated for each E-line distance and for each observer group; these descriptive statistics were calculated using software that we 
developed using MATLAB (The MathWorks Inc., Natick, MA). Similarly, the software calculated the proportions in each group suggesting a desire for treatment. Data were exported to a spreadsheet and tables prepared using Excel (Microsoft Corporation, USA). Additionally, graphs were prepared using Minitab version 19 (Minitab Inc., USA).

\section{Results}

\section{Reliability Analysis}

Table 2 shows the first and third quartile rankings of the Likert score. The median interquartile range for all three groups individually was 1 with the maximum interquartile range of 2 . The results indicate that there was generally good agreement for the patients, lay people and clinicians.

\section{Perceived attractiveness of images}

Figure 3 shows median attractiveness ratings assessed by the three observer groups in comparison with E-line distance. Table 3 shows the median data in rank order from most attractive to least attractive with ratings on a Likert scale from 1 to 7 where 1 indicates 'extremely unattractive' and 7 indicates 'extremely attractive'. As can be seen in Figure 3, the overall variation in attractiveness in relation to E-line distance is similar between the observer groups. A lip to E-line distance within the ranges of -12 to $-16 \mathrm{~mm}$ and 0 to $+4 \mathrm{~mm}$ was associated with a reduction in the median attractiveness scores to below 4 in the groups of observers, except for the lay group where the corresponding ranges were -14 to $-16 \mathrm{~mm}$ and +2 to $+4 \mathrm{~mm}$. The patients and lay groups have the same median attractiveness score for the identical images (DH and EG) and for the clinician group there was just one-point difference thus again indicating good repeatability.

\section{Desire for treatment}


Figure 4 demonstrates the proportion expressed as a percentage of each observer group suggesting that treatment is required. The results indicate that clinicians were generally least likely to suggest treatment for varying levels of lips to E-line distance. Images DH and EG were the same, and so repeatability of the 35 clinicians' assessment appears good with two clinicians suggesting treatment for one of the images and three clinicians suggesting treatment for the other image. For the 75 lay people the assessment of the two repeated images showed some variation (15 and $24 \%$ ) however, for the group of 75 patients there are similar proportions 33 and 35\%. For a number of the images there was generally reasonable agreement among clinicians and lay people as to whether treatment is required. Taking 50\% (i.e. majority) of each observer group as a cut-off where the majority suggested treatment, for the lay and clinician groups the only categories for desire for treatment were at a lip to E-line distance within the ranges of $-14 \mathrm{~mm}$ to $-16 \mathrm{~mm}$ and $+2 \mathrm{~mm}$ to $+4 \mathrm{~mm}$ and similarly for the patient groups within the ranges of $-14 \mathrm{~mm}$ to $-16 \mathrm{~mm}$ and $0 \mathrm{~mm}$ to $+4 \mathrm{~mm}$.

For observers who considered attractiveness to be important $(>2$ in the range 1 very unimportant to 4 very important), table 4 indicates the proportions suggesting treatment which are similar to those for the whole group shown in figure 4 . For patients $68 / 75$, for laypeople 71/75, and all clinicians considered attractiveness to be important. Thus taking $50 \%$ as a level for suggesting treatment the lay and clinician groups considered this to apply for a lip to E-line distance within the ranges of -14 to $-16 \mathrm{~mm}$ and +2 to $+4 \mathrm{~mm}$; for the patient group the corresponding values were within the ranges -12 to $-16 \mathrm{~mm}$ and 0 to +4 $\mathrm{mm}$.

\section{Discussion}


The lips are an important facial aesthetic unit and their morphology, volume, degree and proportion of vermilion exposure are all important aesthetic parameters. However, lip prominence, particularly in profile view, is a potentially significant aesthetic parameter.

In terms of perceived attractiveness, the results of this investigation demonstrate that a lip to E-line distance within the ranges of -12 to $-16 \mathrm{~mm}$ and 0 to $+4 \mathrm{~mm}$ was associated with a reduction in the median attractiveness scores to below 4 in the patient and clinician groups of observers; for the lay group, the corresponding range was -14 to $-16 \mathrm{~mm}$ and +2 to $+4 \mathrm{~mm}$. The most attractive images were deemed to be BE and CF (lip to E-line distances - $6 \mathrm{~mm}$ and $4 \mathrm{~mm}$ respectively) by all the observer groups, and relative lip prominence generally appears to be viewed as more attractive than retrusion.

In terms of desire for treatment the results indicate that clinicians were generally least likely to suggest treatment for varying levels of lips to E-line distance. For a number of the images there was generally reasonable agreement among clinicians and lay people as to whether treatment is required. In the lay and clinician groups desire for treatment was suggested at a lip to E-line distance within the ranges of $-14 \mathrm{~mm}$ to $-16 \mathrm{~mm}$ and $+2 \mathrm{~mm}$ to $+4 \mathrm{~mm}$ and for the patient groups within the ranges of $-14 \mathrm{~mm}$ to $-16 \mathrm{~mm}$ and $0 \mathrm{~mm}$ to $+4 \mathrm{~mm}$.

Previous investigations by Coleman et al. ${ }^{9}$ and Czarnecki et al. ${ }^{10}$ found relatively fuller lips as being more attractive. Their findings are similar to this study as relative bilabial prominence was found to be viewed as more attractive than bilabial retrusion. However, Buschang et al. ${ }^{24}$ have argued that the E-line, and a number of other profile reference lines used to assess lip prominence, may provide erroneous results that do not reflect the actual linear sagittal changes in the position of the lower lip.

Ethnic and gender variation remain an important parameter in attractiveness research studies. In an interesting investigation evaluating ethnic differences in perceptions of attractiveness, Nomura et al. ${ }^{25}$ looked at the perceived attractiveness of varying lip 
prominence relative to the E-line, from $-8 \mathrm{~mm}$ to $+4 \mathrm{~mm}$. Their results demonstrated that preferred lip position, in terms of millimeter distance to the E-line, were $-2.58+/-1.92 \mathrm{~mm}$ for European Americans observers, 3.28 +/- 2.26 mm for Hispanic Americans observers, $3.45+/-1.92 \mathrm{~mm}$ for Japanese observers and -2.13 +/- $1.95 \mathrm{~mm}$ for African observers. They concluded that that lay observer ethnicity and image ethnicity and sex contributed significantly to the judgements of the attractiveness of bilabial prominence. In terms of gender variation, Hier et al. ${ }^{26}$ evaluated the aesthetic preferences of males and females in relation to lip prominence and found that females generally preferred more prominent lips than males, and also that both males and females preferred greater lip prominence than the original Ricketts values. $\mathrm{Hsu}^{27}$ compared five different analyses for lip prominence, and concluded that the E-line is important primarily because it makes the clinician evaluate the nose as part of the facial profile.

The ethnic and gender variation of observers was not a significant factor in this investigation, however, a limitation is that the images were based on a white male Northern European profile. Therefore, it would be prudent for further data to be collected regarding female profiles and those of different ethnic groups.

The data from this investigation has practical implications for the orthodontist, orthognathic surgeon and any clinician contemplating an increase in the soft tissue volume and thereby prominence of a patient's lips. Whether contemplating lip augmentation, changing the inclination or sagittal position of the incisors and thereby their potential support of the lips, particularly in patients with thinner lips, or sagittal movement of the maxilla and/or mandible, the change in lip prominence and the potential effect on facial attractiveness must be considered. The results of this investigation may help guide such decision making.

It should be noted that the images viewed by the observers were based on a young adult male Caucasian profile. As such, the results may not be directly applicable for female 
patients or different ethnic backgrounds. Further investigations may be required to assess variations for such population groups, using both silhouettes and clinical photographs.

\section{Conclusions}

- In terms of perceived attractiveness, a lips to E-line distance within the ranges of $12 \mathrm{~mm}$ to $-16 \mathrm{~mm}$ and $0 \mathrm{~mm}$ to $+4 \mathrm{~mm}$, in relation to the E-line, was associated with a reduction in the median attractiveness scores in the patient and clinician groups of observers; for the lay group the corresponding range was within the ranges of $-14 \mathrm{~mm}$ to $-16 \mathrm{~mm}$ and $+2 \mathrm{~mm}$ to $+4 \mathrm{~mm}$.

- Relative lip prominence appears to be viewed as more attractive than lip retrusion.

- Clinicians were generally least likely to suggest treatment for varying levels of lip position.

- For the clinician and lay groups, the only categories for desire for treatment were at a lip to E-line distance in the ranges of $-14 \mathrm{~mm}$ to $-16 \mathrm{~mm}$ and $+2 \mathrm{~mm}$ to $+4 \mathrm{~mm}$.

- It is recommended that the range of normal variability of the prominence of the lips, as well as threshold values of the desire for treatment, be considered in treatment planning.

\section{Declaration of conflicting interests}

The authors declared no potential conflicts of interest with respect to the research, authorship, and/or publication of this article.

\section{Funding}

The authors received no financial support for the research, authorship, and/or publication of this article. 


\section{References}

1. Naini FB. Facial aesthetics: Concepts and clinical diagnosis. Oxford: WileyBlackwell; 2011.

2. Gill DS, Lloyd T, East C, Naini FB. The facial soft tissue effects of orthognathic surgery. Facial Plast Surg 2017;33:519-25.

3. Ricketts RM. Planning treatment on the basis of the facial pattern and an estimate of its growth. Angle Orthod 1957;27:14-37.

4. Ricketts RM. Cephalomeric analysis and synthesis. Angle Orthod 1961;31:141-156.

5. Farkas LG, Kolar JC, Munro IR. Geography of the nose: a morphometric study. Aesthetic Plast Surg 1986;10:191-223.

6. Farkas LG, Kolar JC. Anthropometrics and art in the aesthetics of women's faces. Clin Plast Surg 1987;14:599-616.

7. Riolo M, Moyers RE, McNamara J, Hunter WS. An atlas of craniofacial growth. Ann Arbor, MI: University of Michigan Center for Human Growth and Development; 1974.

8. Bhatia SN, Leighton BC. A manual of facial growth: A computer analysis of longitudinal cephalometric growth data. Oxford: Oxford University Press; 1993.

9. Coleman GG, Lindauer SJ, Tüfekçi E, Shroff B, Best AM. Influence of chin prominence on esthetic lip profile preferences. Am J Orthod Dentofacial Orthop 2007;132:36-42.

10. Czarnecki ST, Nanda RS, Currier GF. Perceptions of a balanced facial profile. Am J Orthod Dentofacial Orthop 1993;104:180-187.

11. Foster EJ. Profile preferences among diversified groups. Angle Orthod. 1973;43:3440. 
12. Hall D, Taylor RW, Jacobson A, Sadowsky PL, Bartolucci A. The perception of optimal profile in African Americans versus white Americans as assessed by orthodontists and the lay public. Am J Orthod Dentofacial Orthop. 2000;118:514-25.

13. McKoy-White J, Evans CA, Viana G, Anderson NK, Giddon DB. Facial profile preferences of black women before and after orthodontic treatment. Am J Orthod Dentofacial Orthop. 2006;129:17-23.

14. Mejia-Maidl M, Evans CA, Viana G, Anderson NK, Giddon DB. Preferences for facial profiles between Mexican Americans and Caucasians. Angle Orthod. $2005 ; 75: 953-958$.

15. Battle Y, Schneider M, Magder L, Pae EK. Disparity in opinion on lip protrusiveness in contemporary African American faces. Korean J Orthod. 2018;48(1):23-29.

16. Huang YP, Li WR. Correlation between objective and subjective evaluation of profile in bimaxillary protrusion patients after orthodontic treatment. Angle Orthod 2015;85(4):690-8.

17. Al Abdulwahhab B, Pani S, Al Khunaizi R, Al Faraj N. Assessment of lip position preferences in a Saudi population using computer imaging. Eur J Esthet Dent. $2013 ; 8: 444-452$.

18. Chong HT, Thea KW, Descallar J, Chen Y, Dalci O, Wong R, Darendeliler MA. Comparison of White and Chinese perception of esthetic Chinese lip position. Angle Orthod 2014;84:246-253.

19. Hockley A, Weinstein M, Borislow AJ, Braitman LE. Photos vs silhouettes for evaluation of African American profile esthetics. Am J Orthod Dentofacial Orthop 2012;141:161-168. 
20. Ioi H, Nakata S, Nakasima A, Counts A. Effect of facial convexity on antero-posterior lip positions of the most favored Japanese facial profiles. Angle Orthod 2005;75:32632.

21. Naini FB, Donaldson ANA, McDonald F, Cobourne MT. Assessing the influence of chin prominence on perceived attractiveness in the orthognathic patient, clinician and layperson. Int J Oral Maxillofac Surg 2012;41:839-46.

22. Farkas LG. (1994) Anthropometry of the attractive North American Caucasian face. In: Farkas LG, editor. Anthropometry of the head and face. 2nd ed. New York: Raven Press; 1994. p. 159-179.

23. Langlois JH, Kalakanis L, Rubenstein AJ, Larson A, HallamM, Smoot M. Maxims or myths of beauty? A meta-analytic and theoretical review. Psychol Bull $2000 ; 126: 390-423$.

24. Buschang PH, Fretty K, Campbell PM. Can commonly used profile planes be used to evaluate changes in lower lip position? Angle Orthod 2011;81:557-563.

25. Nomura M, Motegi E, Hatch JP, Gakunga PT, Ng'ang'a PM, Rugh JD, Yamaguchi H. Esthetic preferences of European American, Hispanic American, Japanese, and African judges for soft-tissue profiles. Am J Orthod Dentofacial Orthop 2009;135(4 Suppl):S87-95.

26. Hier LA, Evans CA, BeGole EA, Giddon DB. Comparison of preferences in lip position using computer animated imaging. Angle Orthod. 1999;69:231-238.

27. Hsu BS. Comparisons of the five analytic reference lines of the horizontal lip position: their consistency and sensitivity. Am J Orthod Dentofacial Orthop. 1993;104:355-360. 


\section{Figure legends}

Figure 1 The sagittal prominence of the lips of the idealized profile image was altered incrementally in relation to the E-line, creating a series of images. The twoletter code assigned to each image and the distance of the lips to the E-line in millimetres shown in this figure are provided on each image for clarity; for data collection the distance of the lips to the E-line was not on the images shown to observers, and the two-letter code was located outside each image on the computer monitor background.

Figure 2 An example of an image viewed by study observers on the monitor during data collection.

Figure 3 Graph showing median attractiveness ratings assessed by the three observer groups in relation to E-line distance. The two images (DH and EG) with an Eline distance of $-2 \mathrm{~mm}$ were identical.

Figure 4 Graph showing the proportion expressed as a percentage of each observer group suggesting that treatment is required versus lips to E-line distance. The two images (DH and EG) with an E-line distance of -2 mm were identical. 
Table legends

Table $1 \quad$ Observer demographics

Table 2 First and third quartile rankings of the Likert Score.

Table 3 Median attractiveness ratings in rank order from most to least attractive (sorted by clinician ranking).

Table 4 Proportion of observers suggesting treatment who considered attractiveness to be important. 